\section{Randomized Phase II Trial of Osimertinib With or Without Local Consolidation Therapy for Patients With EGFR-Mutant Metastatic Non-Small Cell Lung Cancer (NORTHSTAR)}

Principal Investigator: Yasir Elamin, MD

Conditions: Malignant neoplasms of respiratory and intrathoracic organs, non-small cell lung cancer

Institution: The University of Texas MD Anderson Cancer Center

The purpose of this randomized phase II trial is to determine whether local consolidation therapy (LCT; surgery, radiation, or surgery in combination with radiation) after osimertinib can help to control non-small cell lung cancer (NSCLC) compared with continued treatment with osimertinib alone. The safety of this treatment will also be studied. Osimertinib is FDA-approved and commercially available for NSCLC treatment. Treatment with surgery and/or radiation after osimertinib is considered investigational in patients with NSCLC. Up to 143 participants will be enrolled in this multicenter study.

\section{Primary Objective:}

- Determine progression-free survival (PFS) [time frame: start of induction, osimertinib up to 22 months]

\section{Secondary Objectives:}

- Determine time to appearance of new metastases [time frame: start of induction osimertinib to time of development of a new lesion, up to 22 months]

- Assess progression of target lesions [time frame: start of induction osimertinib to time of development of a new lesion, up to 22 months]

- Assess progression of nontarget lesions [time frame: start of induction osimertinib to time of development of a new lesion, up to 22 months]

- Assess adverse events of osimertinib 6 LCT [time frame: start of induction osimertinib to time of development of a new lesion, up to 22 months]

- Determine overall survival (OS) [time frame: start of induction osimertinib to death, up to 22 months]

- Determine whether osimertinib 1 LCT improves PFS compared with osimertinib alone in subgroup of patients with oligometastatic NSCLC (up to 3 metastases) [time frame: induction of osimertinib, up to 22 months]

- Determine whether osimertinib 1 LCT improves OS compared with osimertinib alone [time frame: induction, up to 22 months]

- Determine whether there is a difference in survival outcomes or toxicity by radiation treatment modality (protons vs photons) [time frame: start of radiation therapy, up to 22 months]

Contact: Yasir Elamin, MD • 713-563-2300 •

CR_Study_Registration@mdanderson.org

ClinicalTrials.gov Identifier: NCT03410043
The NCCN Oncology Research Program (ORP) strives to improve the quality of life for patients and reduce cancer-related deaths by advancing cancer therapies through research. Since the program's establishment in 1999, the NCCN ORP has brought millions of dollars in research grants to investigators at NCCN Member Institutions. Research grants are provided to NCCN through collaborations with pharmaceutical and biotechnology companies; these grants are in turn used to support scientifically meritorious cancer research efforts.

NCCN ORP studies typically explore new avenues of clinical investigation and seek answers to important cancer-related questions. All studies are approved and funded through a scientific peer-review process and are overseen by the ORP.

This feature highlights an NCCN study funded through the grant mechanism.

doi: $10.6004 /$ jnccn.2020.0029

For more information on specific trials, including patient selection criteria, use the contact information listed with each study.

For more information on the NCCN ORP, including a complete detailing of the clinical studies currently underway at NCCN Member Institutions, go to www. NCCN.org/clinical_trials/clinicians.asp. 